Marketplace is provided as a service to readers using text and images from the manufacturer, supplier or distributor and does not imply endorsement by Vital. Normal and prudent research should be exercised before purchase or use of any product mentioned.

\section{Supporting denture wearers}

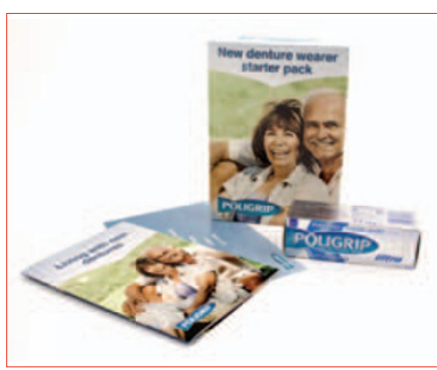

Poligrip has developed a patient support pack for denture wearers. Designed specifically with new denture wearers in mind, the pack includes useful tips and advice on adjusting to new dentures.

Each pack contains a 12page question and answer style information leaflet, a sample of Poligrip denture fixative cream and a 50p coupon for money off your next purchase.

Denture packs can be requested from your GlaxoSmithKline Territory Manager or GSK Customer Services on 02080475000.

\section{New footwear choices}

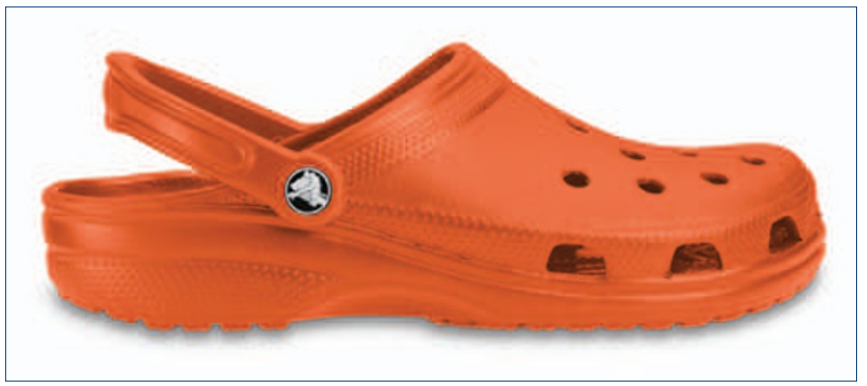

Footwear provider Sweetfeet Shoes are experiencing a steadily increasing number of orders from dental professionals for Crocs shoes. The brightly coloured Crocs Beach and Cayman clog style shoes are comfortable and easy to clean and they have health benefits. Weighing less than six ounces, they have foot massaging nodules which provide an all day massage for hard working feet.

The air vents and foot hugging cushioned non-slip soles keep feet cool, provide good support, and have an excellent grip on every kind of floor surface. Made from a revolutionary odour resistant resin, Crocs can easily be sterilised, making them ideal for working in surgery environments. The resin itself has excellent antibacterial qualities, which help to suppress conditions such as athlete's foot. Sweetfeet Shoes are offering readers a 10\% discount on any pair or pairs of Crocs purchased; just go to www.sweetfeetshoes.co.uk and use the discount code BC02.

\section{Combining nature with science}

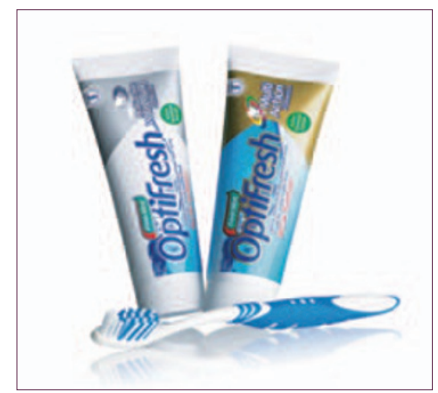

Swedish cosmetics company Oriflame has launched its first toothpaste, Optifresh, to the UK oral care market. Combining nature with science, it contains the Optifresh Advanced Remineralising System, a combination of sea algae (rich in zinc, iron, calcium, and phosphor) that helps remineralise tooth enamel, and fluoride, which protects teeth from acids known to cause cavities and tooth decay.

The range comprises of Optifresh multi-action with a sweet mint flavour and Optifresh multi-action whitening toothpaste with cool water mint flavour. For more information telephone 02074329432 or email oriflame@focuspr.co.uk.

\section{Reducing cross contamination}

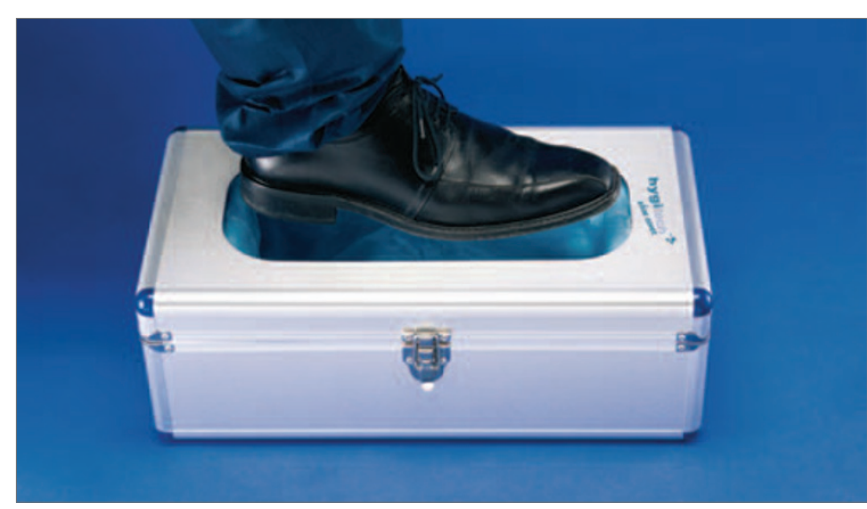

The Hygitech brand from Dental Sky has added three more products to its range.

The Hygitech Shoe Cover Dispenser saves you the trouble of bending down to don shoe covers as well as helping to prevent cross contamination from inadvertently touching dirty footwear.

The Hygitech Spray is a fully automatic, touch-free dispenser for alcohol hand rubs that also assists in the prevention of cross contamination. Without the need to handle anything, the unit automatically detects your hand and dispenses the hydro alcoholic disinfection solution. There is a convenient clear window to allow you to check the level of solution without having to open the front cover.

The Hygitech Perfume Dispenser can also be used to deodorise your surgery, waiting room or rest rooms. With a smart remote control that works up to a distance of 25 metres you can programme the unit to dispense one of the three scents as desired. Choose from lemon, sunrise or elegant scents. For further information or to request your free catalogue contact Dental Sky on 08002944700. 